\title{
Validação de teorias de simulação para operações com escarificadores em solo argiloso no planalto do Estado do Rio Grande do Sul, Brasil
}

\author{
Validation of prediction theories to chisel plow in soils of Rio Grande do Sul State, Brazil
}

\author{
Paula Machado dos Santos ${ }^{\mathrm{I}}$ José Fernando Schlosser $^{\mathrm{II}}$ Alexandre Russini ${ }^{\mathrm{II}}$ André Luís Casali $^{\mathrm{II}}$ \\ Rodrigo Lampert Ribas ${ }^{\mathrm{IV}}$ Orli Luís Roberti Júnior ${ }^{\mathrm{IV}}$
}

\section{RESUMO}

O objetivo deste trabalho foi estudar teorias, utilizadas especialmente para simular situações reais de campo, contrastada por dados empíricos obtidos em condições reais de trabalho agrícola mecanizado em solo de textura argilosa com os resultados obtidos em trabalhos semelhantes realizados em outros países. Avaliou-se as demandas de tração de diferentes combinações entre velocidade e profundidade de trabalho de um escarificador. Buscou-se a representação gráfica da demanda requerida de força de tração e patinagem através da utilização do software Predição versão 1.0, contrastadas com resultados obtidos pela aplicação das teorias de predição Cn, Bn e Mn. As teorias preditivas Cn, Bn e Mn apresentam correlação boa com os valores experimentais, no entanto, existe uma superestimativa por parte das teorias comparadas aos valores medidos experimentalmente, para força de tração e patinagem, causando um dimensionamento do trator inadequado.

Palavras-chave: modelos matemáticos, escarificação, força de tração.

\section{ABSTRACT}

The objective of this research was studied theories used specially to simulate real field situations contrasted by empiric data obtained in real real working conditions in mechanized agricultural clay soil with the results obtained in similar studies conducted in other countries. We tried to the graphical representation of the demand required traction force and slippage through the use of Prediction software version 1.0, contrasted with results obtained by applying the theories of prediction Cn, Bn and Mn. Predictive theories Cn, Bn and Mn have good correlation with experimental values, however, there is an overestimation by the theory compared to the values measured experimentally, for traction force and slippage, causing a tractor design inadequate.

Key words: mathematical models, subsoiling, traction force.

\section{INTRODUÇÃO}

A realização de testes de potência de motores e ensaios em condições reais de trabalho é realizada desde o início da mecanização agrícola, buscando-se conhecer a eficiência de tração dos tratores agrícolas (LINARES et al., 2006). Sendo consideráveis os progressos obtidos nas últimas décadas, no estudo da tração, explica ZOZ (1997), através do desenvolvimento de critérios para avaliar o desempenho do trator com base nos resultados de ensaio realizados em pista de concreto. Entretanto GABRIEL FILHO (2008) cita a necessidade de maior quantidade de pesquisas para avaliar o desempenho em tração sob condições de campo e, com isso, fornecer informações suficientes para que esse atributo seja estimado.

A partir de então muitos trabalhos têm sido realizados na busca de se conhecer o desempenho das máquinas agrícolas que possuem a função de tracionar os implementos, existindo estudos das relações veículoterreno de forma totalmente teórica, pesquisas que utilizam métodos semi-empíricos e, outras baseadas na resistência a penetração do solo. No entanto, as teorias de predição clássicas são as de Wismer \& Luth (Teoria da ASAE ou Cn), Brixius \& Wismer (DEERE ou Bn) e Gee-Clough (NIAE ou MN).

IUniversidade Regional Integrada das Missões e Alto Uruguai (URI), 99700-000, Erechim, RS, Brasil. E-mail: paulagron@hotmail.com. Autor para Correspondência.

"Departamento de Engenharia Rural, Universidade Federal de Santa Maria (UFSM), Santa Maria, RS, Brasil.

IIIPrograma de Pós-graduação em Engenharia Agrícola, UFSM, Santa Maria, RS, Brasil.

${ }^{\text {IV }}$ Curso de Agronomia, UFSM, Santa Maria, RS, Brasil. 
Entre importantes trabalhos realizados no Brasil pode-se citar SCHLOSSER et al. (2004) que realizaram comparações entre teorias de predição do desempenho da tração de trator agrícola, desenvolvidas por Wismer, Brixius e Gee-Clough e concluíram que as teorias são adequadas como ferramenta de simulação, porém, se deve pesquisar diferentes teorias para o uso no meio brasileiro, avaliando-se o modelo que pode adaptar-se mais facilmente e para quais condições de trabalho este é mais preciso.

A determinação de teorias preditivas do desempenho e requerimentos de conjuntos mecanizados para as condições regionais pode servir para otimizar a demanda energética e a combinação entre trator-implemento. Para isso objetivou-se neste trabalho estudar teorias de simulação utilizadas em operações de escarificação contrastada por dados empíricos obtidos em condições reais de trabalho em solo de textura argilosa no planalto do Rio Grande do Sul com os resultados obtidos em trabalhos semelhantes realizados em outros países.

\section{MATERIAL E MÉTODOS}

O trabalho foi conduzido em duas fases distintas. Na primeira, realizou-se um experimento de campo com condições preestabelecidas, quando foram medidas as diferentes variáveis de comportamento do trator, sendo elas o patinamento das rodas dianteiras e traseiras e a força de tração. Na segunda, realizou-se a simulação de comportamento do escarificador com base nas mesmas condições do trabalho de campo, por meio das três diferentes teorias de predição: $C n$, $B n$ e $M n$.

O experimento foi conduzido em solo classificado como Latossolo Vermelho Distrófico típico, plano e bem drenado.

Utilizou-se um escarificador da marca Jan, modelo Jumbo Matic, composto por cinco hastes, rolo destorroador/nivelador e regulagem da profundidade de trabalho através da articulação das rodas, acionado pelo sistema hidráulico de controle remoto do trator; trator marca Massey Ferguson, modelo MF 292RA/ $16 \times 8$ Advanced, com potência bruta no motor de $105 \mathrm{CV}$ a 2.200rpm, os pneus utilizados foram da designação 14.9-26 R1 e 23.1-30 R1, com a utilização de lastros metálicos dianteiros, somando $45 \mathrm{~kg}$.

Visando à obtenção da força de tração requerida pelo implemento, utilizou-se uma célula de carga, marca Alfa, com capacidade de 5.000 a $10.000 \mathrm{~N}$. Como instrumentação eletrônica para aquisição de dados utilizou-se de sensores indutivos de rodas modelo LM12-3004PC e receptor de sinais de satélites marca Trimble, pelo sistema de barra de luzes para determinação da patinagem e um fluxômetro marca Oval (LSN4IL8-M2), modelo Flowmate oval-M III, o qual é composto por duas engrenagens onde uma delas possui um ímã que sensibiliza um sensor indutivo a cada volta ( $1 \mathrm{ml}$ de volume deslocado) para determinar o consumo de combustível, sendo montada no trator de teste e os valores obtidos pelos equipamentos eletrônicos que constituem o sistema de sensores para medir a patinagem que eram conduzidos a um sistema de armazenamento de dados baseado em um datalogger modelo CR-1.000. Posteriormente esses dados eram transferidos para um microcomputador utilizando o software específico para este fim PC400W ${ }^{\circledR}$.

Foram demarcadas 36 parcelas com comprimento de $30 \mathrm{~m}$ e largura de $3,0 \mathrm{~m}$, onde o conjunto trator-implemento foi deslocado, e obtidas as variáveis respostas. Durante as avaliações, o trator foi utilizado com a rotação do motor em 1.900rpm e usadas quatro velocidades de deslocamento, alterando-se a marcha na caixa de câmbio, sendo elas: $2^{\underline{a}}$ reduzida-baixa, $2^{\mathrm{a}}$ reduzida-alta, $3^{\mathrm{a}}$ reduzida-baixa e $3^{\mathrm{a}}$ reduzida-alta. A amplitude da profundidade de trabalho do escarificador foi determinada pela quantidade utilizada de calços limitadores de curso, localizados nas hastes dos dois cilindros hidráulicos para esta finalidade, obtendo-se profundidades de 20,25 e $30 \mathrm{~cm}$.

Para medição da patinagem (d) das rodas traseiras foram utilizados quatro sensores indutivos, que medem a velocidade teórica das rodas $(\mathrm{Vt}),\left(\mathrm{km} \mathrm{h}^{-1}\right)$ e a velocidade real de deslocamento $(\mathrm{Vr}),\left(\mathrm{km} \mathrm{h}^{-1}\right)$ medida por meio do receptor de sinais de satélites (GPS), da marca Garmim, modelo GPSmap 60CS, sendo calculada da seguinte forma: $\mathrm{d}=[(\mathrm{Vt}-\mathrm{Vr}) / \mathrm{Vt}] \mathrm{x} 100$. A velocidade teórica das rodas foi medida através da instalação de sensores de rotação nas rodas motrizes, composto por duas rosetas com dez pinos cada que são fixadas pelo lado de fora do aro dos pneus através de suportes. Cada roseta possuía um sensor de pulsos onde cada pino da roseta ao passar pelo sensor contava um pulso, e ao final de dez pulsos contava uma volta completa da roda, a qual foi determinada mediante a expressão $\mathrm{Vt}=3,6 \mathrm{x} \omega \mathrm{xr}$, sendo: $\omega$ a velocidade angular da roda; $r$ é o raio dinâmico da roda (condição de referência sob piso de concreto $\mathrm{m}$ ); 3,6 é o fator de conversão para se obter $\mathrm{km} \mathrm{h}^{-1}$.

A resistência à penetração do solo foi medida por um penetrômetro marca Falker, modelo penetroLOG PLG1020, com aquisição de dados a cada $5 \mathrm{~cm}$, acompanhada de coletas de amostras para o teor de água do solo com uma sonda TDR, até a profundidade de $30 \mathrm{~cm}$. 
A deflexão dos pneus varia conforme o peso que gravita sobre a roda e a pressão interna do pneu. A maneira como se realizaram as medidas estáticas, estava de acordo com a norma ASAE S296.3 (1995). A caracterização dos pneus nas simulações é feita pelas suas dimensões.

Para comparar o desempenho das diferentes teorias, utilizou-se o procedimento operacional para a predição baseado na rotina do Programa Informático "Predição" versão 1.0, desenvolvido pelo Núcleo de Ensaios de Máquinas Agrícolas para previsão da tração, coeficiente de força tangencial e patinagem das rodas do trator.

Do trator, é necessário informar ao software o peso estático total, sua distribuição sobre os eixos, dados dimensionais (altura do ponto de engate e a distância entre eixos) e a carga na barra de tração. Além da condição estática, também se determinou as condições dinâmicas de distribuição do peso, isto é, considerando a transferência de peso do eixo dianteiro para o traseiro durante o deslocamento do trator.

Para a comparação entre o previsto nas teorias e o obtido no campo, foram aplicados aos modelos os valores medidos no experimento, possibilitando com estes resultados realizar a análise de correlação entre os dados de simulação e o experimental para as variáveis força de tração e patinagem.

Os dados obtidos na experimentação foram submetidos à análise estatística com o auxílio do programa estatístico Assistat. Calculou-se o coeficiente de correlação de Pearson e determinou-se a significância das diferenças entre os dois métodos de desempenho (experimental e simulado) pelo teste $t$ pareado, a fim de verificar se os valores medidos no campo e simulados têm diferenças significativas, em nível de significância de 5\%.

\section{RESULTADOS E DISCUSSÃO}

As regressões obtidas para o conjunto trator-escarificador apresentaram efeito significativo para a interação entre a velocidade de deslocamento e a profundidade de trabalho. As equações quadráticas foram de elevada eficiência no ajuste dos dados ao modelo com a maioria dos coeficientes de determinação de 0,95 (Tabela 1). Na estimativa dos valores de patinagem verifica-se que seus coeficientes de determinação foram maiores (Tabela 1), ou seja, melhor ajuste dos modelos aos dados, pois estes valores são estimados através de simulações, que tem em suas
Tabela 1 - Coeficientes de regressão para determinação da força de tração e patinagem do conjunto trator-escarificador trabalhando em solo de textura argilosa.

\begin{tabular}{|c|c|c|c|c|}
\hline \multirow{2}{*}{ Variável } & \multirow{2}{*}{ Modelo } & \multicolumn{2}{|c|}{$\begin{array}{l}\text { Coeficientes de } \\
\text { regressão }\end{array}$} & \multirow{2}{*}{$\mathrm{R}^{2}$} \\
\hline & & Símbolo & Valor & \\
\hline \multirow{18}{*}{ Força de Tração } & \multirow{5}{*}{$\begin{array}{l}\text { Medido } \\
\text { experimentalmente }\end{array}$} & $\mathrm{C}_{0}$ & 17.680 & \multirow{5}{*}{0,96} \\
\hline & & $\mathrm{C}_{1}(\mathrm{~S})$ & 3.843 & \\
\hline & & $\mathrm{C}_{2}\left(\mathrm{~S}^{2}\right)$ & -347 & \\
\hline & & $\mathrm{C}_{3}(\mathrm{p})$ & -1.226 & \\
\hline & & $\mathrm{C}_{4}\left(\mathrm{p}^{2}\right)$ & 39 & \\
\hline & \multirow{5}{*}{ Teoria Cn } & $\mathrm{C}_{0}$ & 19.737 & \multirow{5}{*}{0,95} \\
\hline & & $\mathrm{C}_{1}(\mathrm{~S})$ & 3.714 & \\
\hline & & $\mathrm{C}_{2}\left(\mathrm{~S}^{2}\right)$ & $-369,78$ & \\
\hline & & $\mathrm{C}_{3}(\mathrm{p})$ & -1.148 & \\
\hline & & $\mathrm{C}_{4}\left(\mathrm{p}^{2}\right)$ & 38 & \\
\hline & \multirow{5}{*}{ Teoria Bn } & $\mathrm{C}_{0}$ & 20.439 & \multirow{5}{*}{0,95} \\
\hline & & $\mathrm{C}_{1}(\mathrm{~S})$ & 3.874 & \\
\hline & & $\mathrm{C}_{2}\left(\mathrm{~S}^{2}\right)$ & -349 & \\
\hline & & $\mathrm{C}_{3}(\mathrm{p})$ & -123 & \\
\hline & & $\mathrm{C}_{4}\left(\mathrm{p}^{2}\right)$ & 40 & \\
\hline & \multirow{5}{*}{ Teoria Mn } & $\mathrm{C}_{0}$ & 20.347 & \multirow{5}{*}{0,95} \\
\hline & & $\mathrm{C}_{1}(\mathrm{~S})$ & 3.776 & \\
\hline & & $\mathrm{C}_{2}\left(\mathrm{~S}^{2}\right)$ & -340 & \\
\hline \multirow{21}{*}{ Patinagem } & & $\mathrm{C}_{3}(\mathrm{p})$ & -1.178 & \\
\hline & & $\mathrm{C}_{4}\left(\mathrm{p}^{2}\right)$ & 38 & \\
\hline & \multirow{5}{*}{$\begin{array}{l}\text { Medido } \\
\text { experimentalmente }\end{array}$} & $\mathrm{C}_{0}$ & 23,00 & \multirow{5}{*}{0,77} \\
\hline & & $\mathrm{C}_{1}(\mathrm{~S})$ & $-1,53$ & \\
\hline & & $\mathrm{C}_{2}\left(\mathrm{~S}^{2}\right)$ & 0,40 & \\
\hline & & $\mathrm{C}_{3}(\mathrm{p})$ & $-0,90$ & \\
\hline & & $\mathrm{C}_{4}\left(\mathrm{p}^{2}\right)$ & 0,02 & \\
\hline & \multirow{4}{*}{ Teoria Cn } & $\mathrm{C}_{0}$ & $-9,29$ & \multirow{4}{*}{0,78} \\
\hline & & $\mathrm{C}_{1}(\mathrm{~S})$ & $-0,24$ & \\
\hline & & $\mathrm{C}_{2}\left(\mathrm{~S}^{2}\right)$ & 0,07 & \\
\hline & & $\mathrm{C}_{3}(\mathrm{p})$ & 0,75 & \\
\hline & \multirow{5}{*}{ Teoria Bn } & $\mathrm{C}_{0}$ & 35,30 & \multirow{5}{*}{0,93} \\
\hline & & $\mathrm{C}_{1}(\mathrm{~S})$ & 5,9 & \\
\hline & & $\mathrm{C}_{2}\left(\mathrm{~S}^{2}\right)$ & $-0,52$ & \\
\hline & & $\mathrm{C}_{3}(\mathrm{p})$ & $-3,85$ & \\
\hline & & $\mathrm{C}_{4}\left(\mathrm{p}^{2}\right)$ & 0,10 & \\
\hline & \multirow{5}{*}{ Teoria Mn } & $\mathrm{C}_{0}$ & 76,23 & \multirow{5}{*}{0,87} \\
\hline & & $\mathrm{C}_{1}(\mathrm{~S})$ & 10,56 & \\
\hline & & $\mathrm{C}_{2}\left(\mathrm{~S}^{2}\right)$ & $-0,92$ & \\
\hline & & $\mathrm{C}_{3}(\mathrm{p})$ & $-8,74$ & \\
\hline & & $\mathrm{C}_{4}\left(\mathrm{p}^{2}\right)$ & 0,21 & \\
\hline
\end{tabular}

deduções matemáticas coeficientes específicos para este ajuste, enquanto a patinagem medida experimentalmente apresentou o menor coeficiente de determinação, pois este parâmetro possui um comportamento de maior dispersão entre os dados, sofrendo a interferência de resistências ao deslocamento no campo, diferentemente de teorias preditivas. Bem como, observou-se a tendência em teorias de predição de superestimarem a força de tração requerida pelo escarificador (Tabela 2), sendo que a 
Tabela 2 - Médias de força de tração requerida pelo escarificador, patinagem e análise de correlação (r) entre valores medidos experimentalmente e valores simulados pelas teorias de predição para solos de textura argilosa.

\begin{tabular}{|c|c|c|}
\hline Modelos & Médias & $\mathrm{r}$ \\
\hline Experimental & $22.312,09$ & - \\
\hline Teoria Cn & $25.034,76$ & 0,99 \\
\hline Teoria Bn & $25.300,13$ & 0,99 \\
\hline Teoria Mn & $25.317,22$ & 0,99 \\
\hline Experimental & 17,81 & - \\
\hline Teoria Cn & 9,53 & 0,61 \\
\hline Teoria Bn & 19,38 & 0,30 \\
\hline Teoria Mn & 22,61 & 0,19 \\
\hline
\end{tabular}

teoria $\mathrm{Cn}$ apresenta menor diferença de resultados que as demais em relação a medida em campo (Figura 1).

Ao realizar simulação de dados de patinagem pela aplicação da teoria $C n$, SCHLOSSER et al. (2004) observaram uma tendência em subestimar os valores de patinagem, semelhantemente ao valores encontrados neste trabalho, além dos valores experimentais serem explicados em baixo grau de correlação, abaixo de 60\% (Figura 2). Possivelmente a utilização de coeficientes determinados em condições de trabalho européias, onde foram levantados os dados para a elaboração destas teorias sejam os fatores que interferem na qualidade de seu uso para trabalhos realizados no Brasil.

Ao aplicar o teste $\mathrm{t}$ verificou-se a aceitação da hipótese alternativa, ou seja, rejeita-se a hipótese de nulidade da heterogeneidade entre as teorias de simulação e os resultados coletados em para as variáveis força de tração e patinagem, permitindo concluir que os valores medidos experimentalmente e os preditivos pelas teorias $\mathrm{Cn}, \mathrm{Bn}$ e $\mathrm{Mn}$ diferem significativamente, com $5 \%$ de probabilidade de erro. Além da magnitude dos valores serem diferentemente significativos, o comportamento da patinagem não foi o mesmo, apesar de todos os modelos ajustados, tanto

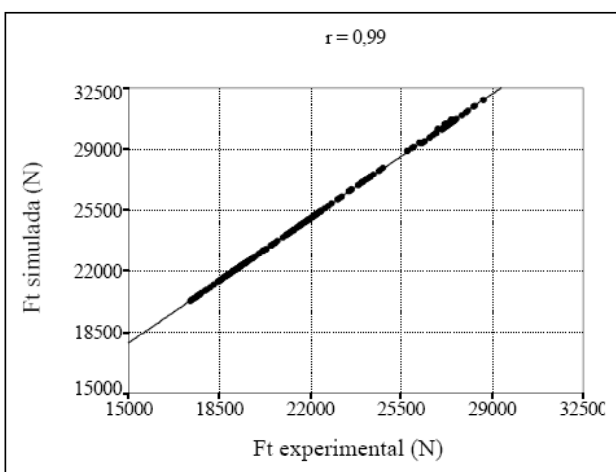

(a)

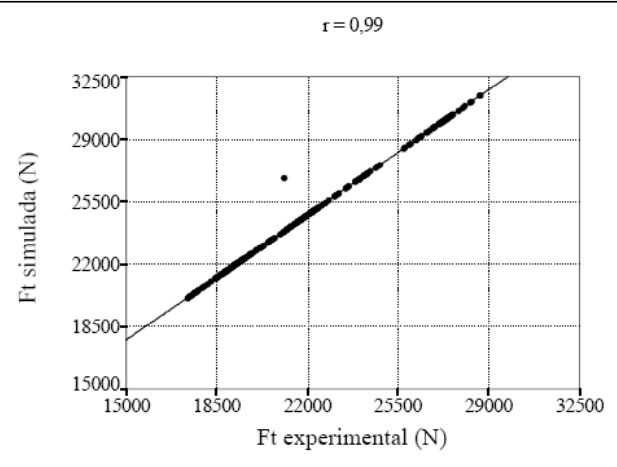

(b)

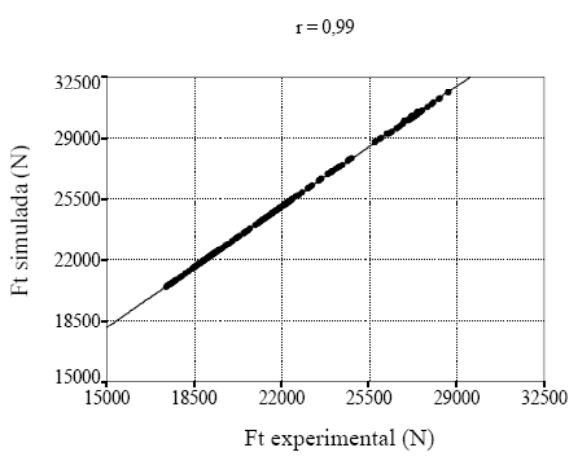

(c)

Figura 1 - Diagrama de dispersão da força de tração medida experimentalmente (Ftexperimental) em função da simulação pelas teorias $\mathrm{Cn}$ (a), Bn (b) e $\mathrm{MN}$ (c) (Ftsimulada) e reta de regressão linear estimada do conjunto trator-escarificador. 


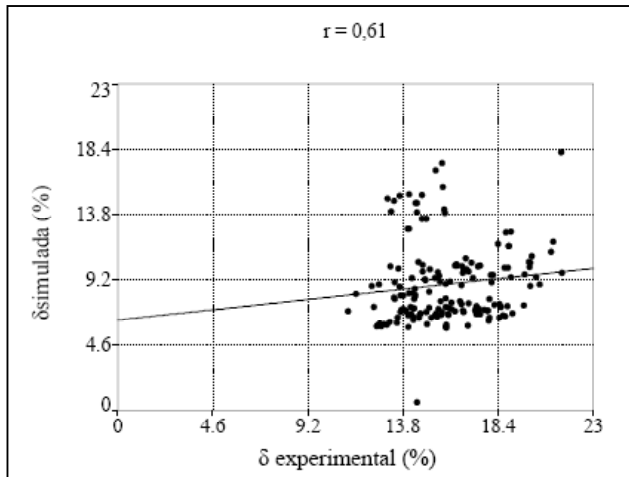

(a)

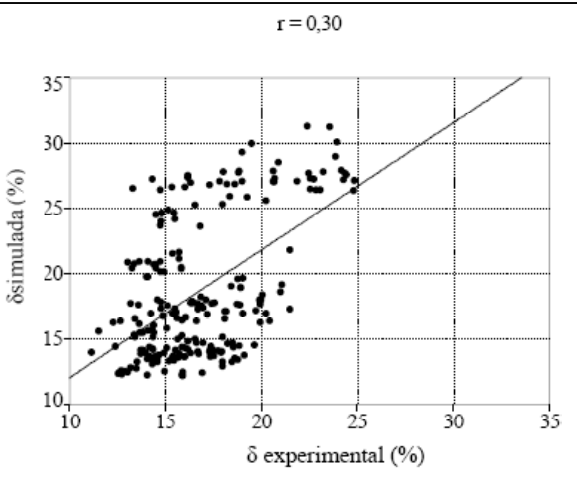

(b)

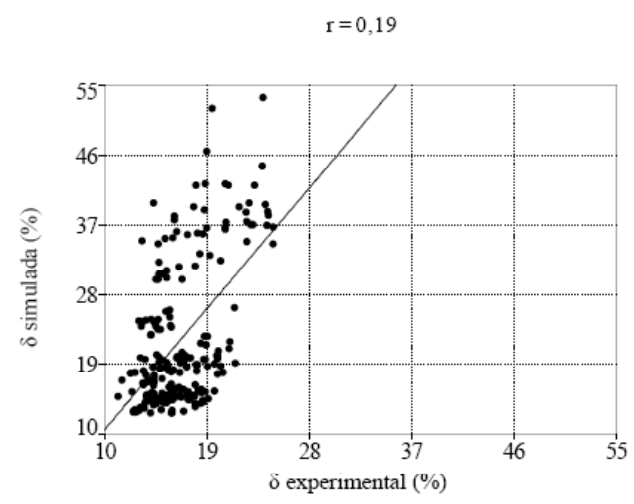

(c)

Figura 2 - Diagrama de dispersão da patinagem medida experimentalmente ( $\delta$ experimental) em função da simulação pelas teorias $\mathrm{Cn}(\mathrm{a}), \mathrm{Bn}(\mathrm{b})$ e $\mathrm{MN}(\mathrm{c})(\delta$ simulada $)$ e reta de regressão linear estimada do conjunto trator-escarificador.

experimentais, quanto os simulados para patinagem apresentarem modelos quadráticos, com coeficientes de determinação adequados, sendo que a patinagem experimental varia em maior magnitude em função da velocidade de deslocamento que os demais modelos estimados pelas teorias (Figura 3), pois apresentaram maior variação com o aumento da profundidade de trabalho.

De acordo com os resultados verificados neste trabalho, SCHLOSSER et al. (2004) observaram que a teoria $C n$ não obteve bons resultados de simulação, desempenho este que confirma a sensibilidade aos valores de índices de cone, conforme citado por CERVANTES (1993). Já as teorias $B n$ e $M N$, menos sensíveis ao efeito solo que a anterior, apresentando resultados que, na maioria das condições, não diferiram significativamente, com tendência de superestimação dos valores nas patinagens mais altas. Esta teoria utiliza em sua simulação valores de índices relacionado à características da roda maior que valores para do solo, como a resistência ao rolamento.

\section{CONCLUSÃO}

As teorias de predição propostas por Wismer e Luth (Cn), Brixius e Wismer (Bn) e GeeClough $(\mathrm{Mn})$ foram diferentes na determinação dos parâmetros força de tração e patinagem, diferindo significativamente dos resultados obtidos experimentalmente, demonstrando tendências de superestimação dos mesmos ocasionando um dimensionamento do trator superior ao realmente necessário para a operação de escarificação.

Embora, o comportamento dos modelos simulados comparado ao experimental seja fortemente correlacionado para a força de tração, apresentando o mesmo comportamento para esta variável quando se alteram a velocidade de deslocamento juntamente com a profundidade de trabalho, existe diferença nas 


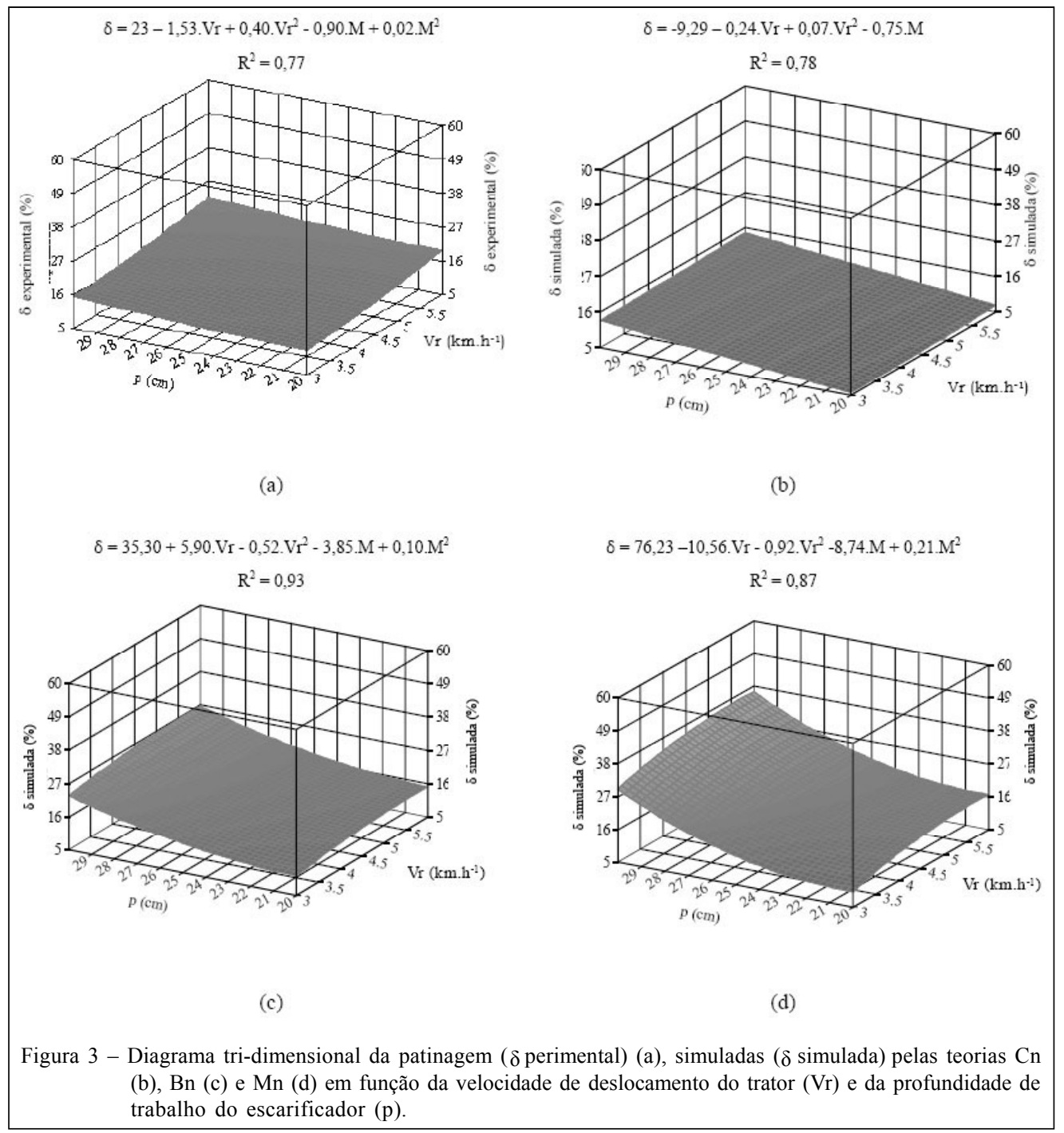

magnitudes entre as teorias $\mathrm{Cn}$, Bn e $\mathrm{Mn}$ em relação aos valores medidos em campo, sendo estes menores aos simulados.

\section{REFERÊNCIAS}

ASAE STANDARD. Uniform terminology for traction of agricultural tractors, self-propelled implements, and other traction and transport device. St. Joseph: American Society of Agricultural Engineering, 1995. p.119-21. (ASAE Standard: S296.3)

BRIXIUS, W.W.; WISMER, R.D. Traction prediction equations for wheeled vehicles. Project 9008. Moline, Illinois: Technical Center, Deere\&Company, 1975. (John Deere Report n.109).

CERVANTES, V.M. Estudio experimental del sistema suelo-vehículo aplicado a la predicción de tracción de los tractores agrícolas. 1993. 143f, Tomo I. 275f, Tomo II. Tese (Doutorado em Energia, Máquinas e Irrigação) - E.T.S.I Agrónomos de Madrid, Madrid, Espanha.

GABRIEL FILHO, A. et al. Umeb - unidade móvel para ensaio da barra de tração. Engenharia Agrícola, Jaboticabal, v.28, n.4, p.782-789, 2008. Disponível em: <http://www.scielo.br/ s c i e lo.php? script=sci_art text \& pid=S 0100 $69162008000400018 \& \operatorname{lng}=\mathrm{en} \& \mathrm{nrm}=\mathrm{iso}>$. Acesso em: 12 abr. 2009. doi: 10.1590/S0100-69162008000400018.

GEE-CLOUGH, D. et al. Tractive performance of drive tyres. Journal of Agricultural Engineering Research, London, v.22, n.4, p.373-404, 1978. Disponível em: <http:// w w w . s c i e n c e d i r e c t . c o m / science?_ob=ArticleURL\&_udi=B6WH1-494T5V0$6 \mathrm{~K} \&$ _user $=10 \& \_$coverDate $=12 \% 2 \mathrm{~F} 31 \% 2 \mathrm{~F} 1977 \& \_$rdoc $=1 \& \_\mathrm{fmt}=\mathrm{h}$ igh\&_orig $=$ search\&_origin $=$ search\&_sort $=\mathrm{d} \&$ docanchor $=\& \mathrm{vi}$ $\mathrm{ew}=\mathrm{c} \&$ _acct $=\mathrm{C} 000050221 \&$ _version $=1 \&$ urlVersion $=0 \&$ _us 
erid=10\&md5=000c5e4c52fc2fc89df56150b4b8ae43\&searchtype $=a>$. Acesso em: 30 out. 2008. doi: 10.1016/0021-8634(77)90070-1.

LINARES, P. et al. Teoría de la tracción en los tractores agrícolas. Madrid: Universidade Politécnica de Madrid, 2006. $157 \mathrm{p}$.

SCHLOSSER, J.F. et al. Desempenho de três teorias de simulação do comportamento de um trator agrícola em tração. Engenharia Agrícola, Jaboticabal, v.24, n.3, p.720-726, 2004. Disponível em:
$<$ http://www.scielo.br/scieloOrg/php/reference.php?pid=S0100$69162004000300024 \&$ caller $=w w w . s c i e l o . b r \& l a n g=e n>$. Acesso em: 11 jun. 2008. doi: 10.1590/S0100-69162004000300024.

WISMER, R.D.; LUTH, H.J. Off-road traction prediction of wheeled vehicles. Transactions of the ASAE, St. Joseph, v.72, n.619, p.15, 1972.

ZOZ, F. Belt and tire tractive performance. Milwaukee: Society of Automotive Engineers, 1997. 8p. 